\title{
Les climats chauds et la production laitière
}

\author{
par M.-G. CURASSON
}

LA question de la production laitière revêt, sous les tropiques, une particulière importance, importance qui est liée à la variété des aspects de cette question et au fait qu'elle interesse à la fois l'hygiène humaine et les industries de l'élevage.

Il n'est pas nécessaire de rappeler que, dans bien des régions tropicales, l'indigène ignore l'usage du lait; que dans d'autres, la consommation est infime, et que cette sous-alimentation azotée est une des principales carences qu'il va falloir combattre dans les régions qui, les plus déshéritées, doivent être les premières à être haussées vers le mieux être que visent à établir les nations européennes tutrices ou associées. Ce manque de lait se fait aussi sentir dans les grandes villes tropicales que n'entoure pas une ceinture d'élevage, ou dont l'évolution trop rapide n'a pas été suivie d'une organisation parallèle de l'hygiène alimentaire. Dans l'un ou l'autre cas, il y a nécessité d'aménager cette production laitiere, et probablement selon des moyens qui varieront avec les circonstances de lieu et de climat.

Un autre aspect de cette question tient au caractere spécial du métabolisme basal chez la vache laitièrc. La labilité particulière de ce métabolisme fait que la vache laitière est un réactif très sensible aux agressions du climat et doit être choisie comme test dans les tentatives d'acclimatement, d'adaptation à des conditions différentes d'alimentation ou de climat.

La diversité du problème laitier tient aussi à la variété dans les populations animales, dans l'état d'évolution des éleveurs, dans les différences climatiques. A la vérité, l'éventail des races qu'il intéresse va de la race demi-sauvage, inexploitée, au produit le plus perfectionné de la zootechnie, tout comme l'éleveur peut aussi bien être le nomade qui vit en bordure de la civilisation que le colon rompu aux règles les plus modernes de l'élevage. On peut faire des remarques du même ordre en ce qui concerne l'habitat, le mode d'entretien, l'alimentation, cette dernière allant de la maigre paille des steppes calcinés par l'été aux grasses nourritures que peut obtenir le colon ou l'éleveur indigène évolué.

Cette complexité ne saurait être exposée en détail.
Dans cette étude d'ensemble, seront d'abord examinées les réactions de la vache aux facteurs climatiques, réactions qui, intervenant sur le métabolisme ou le comportement général, sont à la base de variations quantitatives ou qualitatives, et probablement des différences saisonnières examinées par la suite; ces dernières ont surtout fait l'objet d'études en Europe, mais elles fournissent des indications pour la question examinée. Cela conduit à examiner ensuite les caractères des laits des diverses régions chaudes. Il ne s'agit pas là d'une étude détaillée, mais du choix d'exemples recueillis dans les régions' les plus caractéristiques. Cet examen permet de rechercher ensuite comment, dans ces conditions diverses, le climal peut influencer la production, puis de proposer, à la situation exposée, des palliatifs.

\section{1. - RÉACTIONS DE LA VACHE aUX FACTEURS CLIMATIQUES}

L'expérimentation, dans les recherches relatives à l'acclimatation, comporte des essais réalisés soit "sur le champ », soil dans des conditions artificielles; cette dernière miéthode permet mieux que toute autre de séparer l'action de chaque facteur climatique : chaleur, humidité, vent, etc. Ces expériences sont relativement peu nombreuses et surtout réalisées pour juger du comportement général des animaux à l'égard de ces facteurs. Il en est cependant un certain nombre qui ont eu pour but de rechercher l'influence sur la vache laitière.

Les premières paraissent être celles de Regan et Richardson (1938); elles portaient sur des vaches de races diverses Jersey, Guernesey. Holstein. placées dans une chambre psychrométrique où la chaleur variait, jusqu'à $35^{\circ} \mathrm{C}$. Le tableau ci-dessous résurne les résultats.

\begin{tabular}{|c|c|c|c|c|c|}
\hline $\begin{array}{l}\text { Temp. } \\
\text { atmosp. }\end{array}$ & $\begin{array}{l}\text { temp. } \\
\text { corpo. }\end{array}$ & $\begin{array}{l}\text { rend. } \\
\text { journ. } \\
\text { en livres }\end{array}$ & graisses & $\begin{array}{c}\text { solides } \\
\text { non gras } \\
\mathbf{y}_{0}\end{array}$ & caséine \\
\hline $4^{\circ} 4$ & $37^{\circ} 6$ & 29 & 4,2 & 8,26 & 2,26 \\
\hline $26^{\circ} 6$ & 39 & 25 & 4 & 7,84 & 2,07 \\
\hline $29^{\circ} 4$ & $39^{\circ} 1$ & 23 & 3,9 & 7,68 & 1,93 \\
\hline $32 \cdot 2$ & 3908 & 20 & 4 & 7,64 & 1,91 \\
\hline 35 & $40^{\circ} 6$ & 17 & 4,3 & 7,58 & 1,81 \\
\hline
\end{tabular}




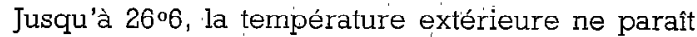
pas avoir d'influence marquée; mais dès que l'hyperthermie est atteinte; il y a baisse de la production journalière; cette baisse est rapide et à $35^{\circ}$ elle atteint $41 \%$. La teneur en matière grasse est peu affectée, mais au-dessus de cette température, il y a des modifications de composition; il y a moins d'acides gras à petite molécule.

A Singapour, des vaches Holstein maintenues pendant les mois d'été dans une étable à air conditionné, la température étant de $21^{\circ} \mathrm{C}$., et le degré hygrométrique de $60 \%$, ont un rendenent. juurnalier de 24 livres alors qu'il n'était que de 9 livres dans un hangar ventilé par l'air chaud (Smith, 1941).

Dans l'Arizona, les expériences de Davis R.-N. et collaborateurs (1947) montrent que la température extérieure joue un rôle important dans la composition du lait. La moyenne établie pour diverses races (Jersey, Guernesey, Holstein) indique que, pour chaque augmentation de $6^{0}$. pour une température moyenne entre 18 et $40^{\circ} \mathrm{C}$., la teneur en beurre diminue de $0,1 \%$. La matière sèche, la graisse, les protéines sont plus innporlantes en hiver qu'en été.

La production laitière est plus faible et de qualité inférieure quand la température est chaude et sèche, même si une nourriture adéquate est maintenue. C'est ce que montrent des observations faites en Californie sur des vaches de race de Jersey ou Holstein. Dans les remarques relatées ci-dessous, la température moyenne, en Juillet, fut de $26^{\circ} \mathrm{C}$., et il n'y eut que dix nuits au cours desquelles elle fut inférieure à $21^{\circ}$. D'autre part, avec des nuits au cours desquelles elle dépassa ce taux, il y a coïncidence d'une baisse sensible de production (Ittner, 1947).

\begin{tabular}{|c|c|c|c|c|c|c|}
\hline \multirow[b]{2}{*}{ Dates } & \multicolumn{3}{|c|}{ HOLSTEIN } & \multicolumn{3}{|c|}{ JERSEY } \\
\hline & $\begin{array}{l}\text { temp. } \\
\text { matin }\end{array}$ & $\underbrace{}_{\begin{array}{c}\text { corpo. } \\
\text { soir }\end{array}}$ & $\begin{array}{c}\text { rendt } \\
\text { par tête } \\
\text { en livres }\end{array}$ & $\begin{array}{l}\text { temp. } \\
\text { matin }\end{array}$ & corpo. & $\begin{array}{l}\text { rendt } \\
\text { pas tête } \\
\text { en livres }\end{array}$ \\
\hline $0 \mathrm{M}$ & $38^{\circ} 3$ & 3805 & 52,9 & 38035 & $38 \circ 6$ & 29,5 \\
\hline Ju & $38 \circ 6$ & $39 \circ 6$ & 48,3 & $38^{\circ} 6$ & $38 \circ 9$ & 27,9 \\
\hline Juillet. . & 3902 & 40001 & 37,2 & $38 \circ 5$ & 3904 & 23,6 \\
\hline Août. & $39 \circ 16$ & $40 \circ 01$ & 30,3 & 3803 & $39 \circ 2$ & 24,4 \\
\hline Sept.. & $39 \circ 1$ & $39 \circ 6$ & 27,9 & $38 \cdot 35$ & 39 & 23,2 \\
\hline Octobr & 8801 & $38^{\circ} 6$ & 22,2 & $38^{\circ} 38$ & 38035 & 19 \\
\hline
\end{tabular}

Ainsi, les deux races sont influencées, mais de façon différente : dans les mêmes conditions de climat, la production est réduite de $58 \%$ chez les Holstein et seulement de $36 \%$ chez les Jerseyaises.

Ce comportement particulier de la race de Jersey est d'ailleurs une illustration de ses facultés spéciales de résistance aux climats chauds, telle qu'elle ressort du mode adopté par Rhoad (1944) pour ramener à une formule la reprćscntation de ce complexe physiologique qu'est la résistance à la chaleur. Pour, cela, l'auteur expérimente en pleine saison chaude, la température à l'ombre évoluant entre 29 et $35^{\circ} \mathrm{C}$, ou plus. Les bovins sont placés dans un enclos provisoire clos de fil de fer, gazonné, peu après le lever du soleil. Ils sont en liberté et ont de l'eau à leur disposition. A 10 heures, on les place doucement sous abri pour prendre la ternpérature rectale et noter le nombrc de respirations; on les remet dans l'enclos pour recommencer les observations à 15 heures. On fait la moyenne de ces deux observations, pour chaque animal. Les animaux tranquilles peuvent être attachés, mais il est important d'éviter la proximité de bâtiments qui peuvent fournir de l'ombre 'ou, au contraire, gêner' les mouvements d'air. Il faut aussi qu'ils soient sur gazon et non sur dú pavé, pour rester dans les conditions naturelles. Les observations faites, la résistance à la chalęur est appréciée par la formule suivante :

$$
\mathrm{RC}=100-[10(\mathrm{TC}-101,1)]
$$

Dans cette formule, T.C. est la température corporelle moyenne obtenue au cours des essais, 101 est la température normale des bovins, 10 est le facteur qui permet de traduire les degrés de variations de température corporelle; 100 indique la perfection, celle de l'animal qui maintient sa température à $101^{\circ} \mathrm{F}$. (3803). Comme exemple, un animal ou un groupe d'animaux qui, au cours de l'expérimentation, a eu une moyenne de $103 \circ 8 \mathrm{~F}$. (39.9) a un coefficient' de résistance égale à :

$100-[10(103,8-101)]=100-[10(2,8)]=100-28=72$

Les resultats obtenus en Louisiane (station expérimentale de Jeanerette), les moyennes étant établies pour des groupes de vaches de même race, 'sont résumés dans le tableau suivant ' (la température extérieure étant en moyenne de $32^{\circ} \mathrm{C}$. le matin de $34^{\circ} \mathrm{C}$. l'après-midi et l'humidité relative de 82 et $71,7 \%)$.

\begin{tabular}{|c|c|c|}
\hline Races & $\begin{array}{l}\text { nombre } \\
\text { de tests }\end{array}$ & $\begin{array}{l}\text { coefficient } \\
\text { de' résistance }\end{array}$ \\
\hline Zébu & 18 & 89 \\
\hline 1/2 Zébu - 1/2 Angus & 67 & 84 \\
\hline 3/8 Zébu - 5/8 Angus & 21 & 84 \\
\hline Santa Gestrudis. . . . & 21 & 82 \\
\hline 1/2 Afrikander - 1/2 Angus... & 64 & 80 \\
\hline Jersey $\ldots \ldots \ldots \ldots \ldots \ldots \ldots$ & 34 & $79:$ \\
\hline 1/4 Zébu - 3/4 Angus & 65 & $77^{\prime}$ \\
\hline Hereford pure $\ldots$ & 12 & 73 \\
\hline 1/4 Afrikander $-3 / 4$ Angus... & 9 & 72 \\
\hline Angus .............. & 69 & 59 \\
\hline
\end{tabular}

A la même station, Gaalas (1947), expérimentant sur des vaches de race Jersey, modifie la formule de Rhoad, pour tenir compte de ce que les vaches 
pouvaient se mettre à l'abri. La formule employée eșt :

$$
\mathrm{RC}=100-[14(\mathrm{TC}-101)]
$$

la température extérieure moyenne étant $32^{\circ} 2 \mathrm{C}$.

Le chiffre moyen est 78,1. Dans le troupeau exáminé, il n'y a pas de différence marquée, concernant la résistance, d'une année à l'autre, mais l'âge intervient, les animaux de deux ans ayant la résistance la plus faible, ceux de trois ans jusqu'à huit ans la plus forte, pour diminuer ensuite. Il y a des différences individuelles; la période de lactation et de gestation a peu ou pas d'effet.

Chez les vaches laitières, il y a une nette différence de comportement à l'égard de la températura au-dessus de $25^{\circ} \mathrm{C}$., différence dont le facteur le plus important est le volume corporel. La température critique, celle qui commence à exercer un effet définitif de dépression, commence à $25-27^{\circ}$ chez les vaches de grand format, et à $27-30^{\circ}$ chez les vaches de petit format. Il peut survenir, chez les bêtes de grand format, qui ont une plus grande température rectale, des cas d'avortement deux ou trois jours après exposition, pendant trente heures à $38^{\circ} \mathrm{C}$. Peut-être y a-t-il mort thermique du fœetus. Chez les grandes vaches également, la teneur en matière grasse, parallèlement à la diminution de la sécrétion lactée, augmente avec la température (Brody, Ragsdale et Thompson, 1948).

Le rendement en lait étant essentiellement lié aux réactions de la vache à l'égard des facteurs chaleur et humidité, il est intéressant de noter les résultats expéfimentaux obtenus à ce dernier titre.

Dég expériences faites en Californie ont montré que, chéz des vaches maintenues à l'étable, la température corporelle reste constamment à 3803-3804 tant que la température de l'étable ne dépasse pas

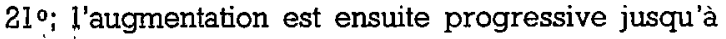
atteindre 4005 quand la température est de $37-38^{\circ}$ (Hornby, 1942).

Le rôle respectif de la température et de l'humidité, en. saison chaude et humide, a été étudié par Seath et Miller (1946) en Louisiane, sur des vaches de races Holstein et Jersey. On observe ainsi qu'une augmentation de la température de $1^{\circ} \mathrm{F}$., augmente la température, corporelle 13 à 15 fois plus, et le rythme respiratoire 41 à 43 fois plus qu'une augmentation de $1 \%$ de l'humidité atmosphérique.

La température extérieure étant de $34^{\circ}$ (en Louisiane), la température corporelle de vaches jerseyaises, après deux heures d'exposition au soleil. est de $39 \circ 3$, et le rythme respiratoire de 83 ; ce dernier tombe à 56 après une heure de retour à l'ombre (Seath et Miller, 1947).

On a comparé, en saison chaude, en 1944 et 1945, la résistance à la chaleur des vaches de race Holstein ou Jersey. La température moyenne fut de $26^{\circ} 8$ en
1944 (maximum $34^{\circ}$ ) et de $27^{\circ}$ en 1945 (maximum $3^{\circ}$ ). La température corporelle moyenne (prise le matin) fut en 1944 de $39^{\circ} 6$ pour les Holstein et $39^{\circ} \mathrm{l}$ pour les Jersey, en 1945, respectivement de $39 \circ 7$ et $39 \circ 1$. Les pulsations et les respirations n'offraient pas de différences; le taux d'augmentation de la température corporelle est plus marqué chez les Holstein. En 1944, une augmentation de l'humidité fut suivie de l'augmentation de la température corporelle et du rythme respiratoire chez les Jersey, pas chez les Holstein. Il n'y a pas de relation apparente entre la proportion de blanc chez les Holstein et la résistance à la chaleur (Seath et Miller, 1947).

Chez des vaches (Holstein-Friese) maintenues à $31^{\circ} \mathrm{C}$., l'humidite relative étant de $60 \%$, le nombre moyen des respirations est en moyenne de 78,5 par minute à l'ombre, dehors, et de 80,5 à l'étable; à l'étable également, la température corporelle était de $39^{\circ} 6$.

Des vaches de race Jersey placées au soleil, alors que la température à l'ombre est de $32^{\circ} \mathrm{C}$., ont une température rectale de $40^{\circ} \mathrm{C}$.; si on les place à l'ombre, la température rectale tombe à $38 \circ 8$, et à $38^{\circ} 2 \mathrm{si}$, à l'ombre, on produit une aspersion d'eau finement pulvérisée. Dans ces conditions différentes, le nombre des respirations est respectivement de 113 , 82 et 56. Des génisses sont moins influencées par la chaleur et ne recourent pas aussi souvent à l'ombre.

Dans d'autres expériences, des vaches de même race, la température extérieure variant de 30 à $36^{\circ} \mathrm{C}$., ont une température rectale moyenne de 3901 (extrêmes $38^{\circ} 4$ et $42^{\circ}$ ) ce qui dépasse de 101 la moyenne normale, et le nombre moyen des respirations est de 109, ce qui est plus du double du nombre observé à une température froide. $\mathrm{Si}$ on asperge et ventile les animaux, la température peut être abaissée de 10 , et le nombre des respirations diminuer de 27 à 42 selon qu'ils sont simplement aspergés ou aspergés et ventilés (Seath et Miller, 1948).

Au Queensland, Riek et Lee (1948) placent des vaches de race de Jersey, deux fois par semaine pendant sept heures à des températures allant de 29 à $43^{\circ} \mathrm{C}$., l'humidité absolue variant de 6 à $16 \mathrm{gr}$. par pied cubique.

La température rectale augmente avec la température atmosphérique, mais ne dépasse pas $42^{\circ}$, la tempéráture extérieure étant alors de $43^{\circ}$ et le degré hygrométrique maximum.

Le rythme respiratoire est affecté parallèlement; comme la température, la respiration est augmentée avec l'humidité; elle peut atteindre 200 mouvements par minute; cependant, le volume d'air inspiré est diminué.

Le nombre des pulsations reste à peu près normal quand la température augmente, mais l'humidité a tendance a le faire plus important. 
La production 'du lait, sa teneur en matière grasse ne paraissent pas influencées (en raison sans doute - de la brièveté des expositions).

A Dar es Salaam (Soudan), les observations suivantes ont été faites sur un troupeau laitier constitué par des animaux de race Friese, de métis et de zébus. les températures étant prises le matin et l'après-midi, les animaux étant au repos dans un hangar couvert après avoir pâturé sous des palmiersí le matin, la température moyenne était de $24^{\circ} \mathrm{C}$. et l'humidité relative de $94 \%$; l'après-midi, la température de $31^{\circ} \mathrm{C}$. et l'humidité de $62 \%$.

Le matin, la température corporelle était sensiblement la même : $38044-39 \circ 05$ chez les zébus et $38^{\circ} 39$ - 38.94 chez les Friese; le soir, la différence est à peine plus marquée : respectivement $38 \circ 55-39^{\circ} 4$ et $38^{\circ} 7-39^{\circ} 07$. Mais il y a de grandes différences individuelles parmi les vaches de race européenne, ce qui devrait servir à guider la sélection (Kendall, 1948).

La résistance particulière des zébus, et leur rôle dans l'amélioration de la production laitière de bien des régions tropicales s'explique par leur comportement à l'égard de la chaleur et de l'humidité; à l'inverse des races européennes, le zébu, l'Afrikander ont des réactions réduites.

Dans l'Inde, la variété Mariana subit une poussée de température au cours de la journée, coincidant avec la température extérieure; et la moyenne de la température corporelle est plus grande pendant les mois chauds; mais ces poussées sont toujours faibles (Minett et Len, 1945).

Les observations de Bonsma, Scholtz et Badenhorst (1940) en Afrique du Sud montrent bien les différences de comportement de l'Afrikander (Bos indicus) et des races européennes ou de leurs croisements sous le climat d'Afrique du Sud

\begin{tabular}{|c|c|c|c|c|c|c|}
\hline \multirow[b]{2}{*}{$\begin{array}{l}\text { Température } \\
\text { à l'ornbre }\end{array}$} & \multicolumn{3}{|c|}{ température corporelle } & \multicolumn{3}{|c|}{ Respirations } \\
\hline & Hlereflord & $\widetilde{\text { Shorithorn }}$ & $\begin{array}{l}\text { Alrikinder' } \\
\text { Shoorlloran }\end{array}$ & Hereford & Shorthori & $\begin{array}{l}\text { Arilkander } \\
\text { Shlouthorn }\end{array}$ \\
\hline 2505 & $39 \circ$ & $39 \circ 3$ & 3808 & 48 & 78 & 40 \\
\hline $32^{\circ 5}$ & $39 \circ 5$ & $41^{\circ} 1$ & 390 & 94 & 121 & 41 \\
\hline 3404 & $39 \circ 6$ & $41^{\circ}$ & 3809 & 98 & 112 & 84 \\
\hline
\end{tabular}

Au Kenya, des remarques du même ordre ont pu être faites (Daubney, 1942) :

\begin{tabular}{|c|c|c|c|c|c|c|c|}
\hline \multirow{2}{*}{\multicolumn{2}{|c|}{$\begin{array}{l}\text { Température humidité } \\
\text { à l'ombre' (Pres. ei multg }\end{array}$}} & \multicolumn{3}{|c|}{ Lempé. corporelle } & \multicolumn{3}{|c|}{ respiration } \\
\hline & & uebu. & ayrshipe & metis & & aysshis & metis \\
\hline 10 à $13^{\circ} \mathrm{C}$. & 604 & $38^{\circ}$ & 3804 & $38 \circ 1$ & 21 & 30 & 27 \\
\hline 19 à $21^{\circ} \mathrm{C}$. & 1106 & $38^{\circ} 7$ & $39 \circ 1$ & 3808 & 29 & 45 & 37 \\
\hline 24 à 2605 & 10 & $38 \circ 7$ & 3905 & 3809 & 35 & 56 & 44 \\
\hline
\end{tabular}

Au Brésil, les observations de Rhoad (1936) montrent aussi la tolérance du zébu et de ses hybrides avec les races européennes.

\section{II. - VARIATIONS SAISONNIÈRES DANS LA COMPOSITION DU LAIT}

Les opinions diffèrent en ce qui concerne l'influence des saisons sur la composition du lait; cependant, de manière générale, on admet en Europe et en Amérique que la teneur en substances solides est plus grande en hiver qu'en été. Hills (1892) pense que la matière grasse varie de façon inverse de celle de la température, ainsi que les autres composants. Divers autres auteurs trouvent "une diminution de la matière sèche en eté, mais pas d'influence marquée sur la graisse (Brody, 1922, Weaver et Mathews, 1928, Houston et Hale, 1932).

Dans un troupeau de 50 Friese anglaises, la majorité des échantillons ' $(80,1 \%)$ étaient inférieurs au standart de $8.5 \%$ en solides hon gras pendant les mois d'hiver puis augmentait à la mise à l'herbe (Lesser, 1932). Pour Mackintosh (1933) la matière grasse suit une courbe qui s'abaisse en 'été, le froid augmentant la teneur en beurre et la chaleur l'abaissant.

Les observations de Davies (1936) amenent l'auteur à conclure que l'été sec peut causer des variations dans la composition du lait, variations dues' aux effets combinés du manque d'eau alimentaire, de l'irritation causée par les insectes, de la sécrétion d'un liquide moins riche en valeur calorique.

- Pour Turner (1936) quand il y a conditions de sécheresse, la matière grasse et les protéines dépassent le taux normal, alors que les chlorures augmentent et que le lactose diminue, bien que la haule ternpérature, qui accompagne généralement la sécheresse, ait un effet opposé sur la matière grasse.

La qualité du lait est affectée, d'après Kay (1937), ' à la fois par la sécheresse, la chaleur et la mauvaise alimentation qui vont en général de pair; on tempère cette action par une meilleure alimentation en concentrés.

Opérant en Hongrie sur des laits de mélange qu'il examinait deux fois par mois pendant quatre ans, Trambicus (1938) constate que le taux des matières solides non grasses diminue chaque année en été, la baisse étant proportionnelle à la hausse de la température.

En ce qui concerne les chlorures et le lactose, les recherches sur l'influence des saisons, sont peu nombreuses. Cependant', d'après Davies (1937) la sécheresse de l'été amène une hausse légère en chlorures et une baisse légère en lactose, alors que pour Jorgenson et Oostergaard (1939) il n'y a pas de variations saisonnières du lactose; l'opinion de Cranfield et collaborateurs (1927) est cependant que toute diminution de la matière sèche non grasse 
en été correspond à une baisse du lactose plutôt que des protéines. Dans toutes ces observations, il ne semble pas que l'on ait suffisamment tenu compte de l'influence que peut avoir la période de la lactation, laquelle peut amener des variations plus grandes que celles provoquées par la saison (Turner, 1936).

En Amérique (Missouri) la température suffirait à expliquer la baisse des matières sèches non grasses observée en été (Herman, 1938), baisse qu'observent également Jacobsen et Walters (1939).

Les recherches faites dans l'Arizona sur des vaches de diverses races, il résulte que la teneur en matière sèche totale, en matière grasse, est plus grande en hiver qu'en été. L'influence de la température extérieure est plus grande que celle du stade de lactation; elle se traduit par une baisse de la teneur en beurre, plus marquée pour certaines races (Guernesey) que pour d'autres (Jersey, Holstein). La teneur en matière grasse diminue de $0,1 \%$ pour chaque hausse de température de $10^{\circ}$ entre $18^{\circ} \mathrm{C}$. et $40^{\circ}$ C. (Davis et collaborateurs, 1947).

Au Brésil, la teneur en matière grasse est plus grande en Avril et Mai et plus basse en Septembre et Novernbre; à l'inverse de ce que pensent Davis et collaborateurs il semble que c'est le stade de la période de lactation qui joue le role le plus important dans les variations de la teneur en matière grasse (Jordao et Assio, 1946).

Encore au Brésil, des vaches de race Simmenthal subissent dans la production laitière des variations saisonnières marquées; le maximum est atteint au milieu et à la fin de la saison des pluies, le minimum en pleine saison sèche, ce qui correspond aux observations précédentes de Rhoad. Ces variations paraissent surtout dues à ce que la nourriture est différente dans les deux saisons, ce qui est le cas dans toutes les régions tropicales et subtropicales. Comme dans les régions tempérées, la production augmente jusqu'à environ 40 à 50 jours après la naissance, pour redescendre ensuite (Carneiro et Lush, 1948).

En Argentine, on observerait un rythme saisonnier dans le taux des matières grasses, le plus fort taux étant en automne et au début de l'hiver, le plus bas au printemps et au commencement de l'été (Labarthe. 1946).

Aux Iles Fidji, les remarques faites à la station expérimentale de Ligatoka semblent indiquer que, en région tropicale, les températures basses, bien qu'elles ne tombent pas au-dessous de $18^{\circ} \mathrm{C}$. entraînent une baisse de la production plus que ne le fait l'absence de pluie. Au cours de la saison sèch $\theta$, la baisse peut être de 48 à $52 \%$ par rapport au maximum. Mais cet effet parait être indirect, et dû surtout à l'alimentation, trop déficiente pour parer à l'action du froid (Sanders, 1948).
En Australie, l'analyse des laits de mélange montre que la matière sèche non grasse baisse en hiver et en été et a son maximum au printemps et à l'automne. Les variations de la matière grasse sont plus marquées, mais moins régulières, et il semble que lorsqu'elle baisse, la matière sèche non grasse monte, et inversement (Rees, 1947).

En Afrique du Sud, d'après Smith (1929), les matieres solides augmentent en hiver et diminuent au printemps et en été, le point le plus bas étant en Février. Quand survient une saison sèche et chaude assez longue, on observe une baisse des matières solides non grasses.

En tenant compte des divers facteurs qui peuvent influencer la composition, et au cours d'expériences prolongées sur des vaches Friesland entretenues en Afrique du Sud, Van Rensburg (1947) arrive à la conclusion que, contrairement à ce qu'on observe en Europe et en Amérique, c'est en hiver que la qualité du lait est moins bonne, le pourcentage de matières sèches non grasses tombant au-dessous du standart $8,5 \%$. La matière grasse et le lactose subissent une baisse similaire, et l'index chlorurelactose augmente parallèlement. Par contre, en été, la matière sèche et le lactose augmentent, l'index chlorure-lactose diminue. Ainsi, on peut observer des différences de l'ordre suivant:

\begin{tabular}{l|cccc} 
& \multicolumn{4}{c}{$\begin{array}{c}\text { Matière sèche } \\
\text { non grasse }\end{array}$} \\
Hiver (Juin) ..... & 50 & 2,3 & 45,6 & 45,6 \\
Eté (Décombre). & 32 & 10 & 14,8 & 14,8
\end{tabular}

Les expériences n'ont pu établir l'influence de la haute température sur l'abaissement de la qualité du lait; au contraire, le meilleur lait fut sécrété pendant les mois les plus chauds. Quant à la baisse de qualité en hiver elle paraît devoir être pour grande part attribuee à l'alimentation.

Dans les régions d'Afrique du Sud comprises dans les zones à pluies d'été ou à pluies d'hiver, on n'a pas observé, au cours d'un même mois, de variations dans la teneur en matière grasse liées à la température. Par contre, dans les régions à pluies d'hiver, il y a une relation marquée entre cette teneur et la température moyenne des quatre mois précédents, alors que dans les régions à pluies d'été, la relation la plus marquée correspond à la température des trois mois précédents. On observe, sans l'expliquer, que la teneur en matière grasse augmente au début de la saison froide, plutôt qu'en pleine saison froide, et qu'elle baisse au début de la saison chaude, plutôt qu'en pleine saison chaude.

Quant à la matière sèche (non grasse), elle augmente au milieu de l'été dans les régions à pluies d'été; dans ces régions, la quantité de lait produite 
est fortement en relation avec la, quantité d'eau tombée, alors que dans les régions à pluies d'hiver, l'augmentation commence à se faire sentir seulement vers la fin de la saison, quand la température commence à remonter. Il n'y a pas de relations entre la teneur en matière grasse et la quantité menstielle de lait dans les, régions à pluies d'été, alors que dans les régions à pluies d'hiver, la teneur a son maximum quand la production est à son maximum (Bakalor, 1948).

De toutes ces observations, il est difficile de tirer des conclusions sûres; c'est que, dans certaines, il n'a pas été tenu suffisamment compte des facteurs qui, en dehors des facteurs climatiques, interviennent dans les variations de la composition du lait. De ces facteurs le plus important, l'alimentation, joue en général son rôle en même temps que la saison; nous verrons, au cours de celle élude, de nombreux exemples qui montrent l'action compensatrice de l'alimentation à l'égard de l'action du climat; il est évident que dans les régions à élevage extensif, où l'animal ne reçoit guère que l'alimentation fournie par le pâturage, la pluviométrie intervient surtout de façon indirecte, pour fournir des fourrages plus aqueux. Ainsi, dans une ferme du Niger, une saison des pluies précoce pendant laquelle il tomba 455 millimètres d'eau, chiffre exceptionnel, permit de noter chez des vaches de race locale une production atteignant 7 litres par jour, ce qui dépassait de beaucoup la production habituelle.

C'est aussi par l'alimentation que la saison peut intervenir, non plus sur la quantité du lait, mais sur sa composition : en Nouvelle-Zélande, en nourrissant des groupes de vaches laitières alternativement avec une ration normale et une ration faible, on remarque que, si la graisse ne diminue pas avec la ration faible, il y a par contre baisse de la matière sèche non grasse (Riddet et collab., 1941).

Des observations faites en régions tempérées, et qui pourraient fournir des indications pour les régions chaudes, montrent par ailleurs que la production laitière est influencée par la saison à laquelle se fait la mise bas. Ainsi, en Norvège, c'est l'automne qui paraît la saison la plus favorable; quand les naissances se font à la fin du printemps et en été, la production est de $20 \%$ plus faible que pour les naissances d'automne, Octobre étant le meilleur mois et Juin le plus mauvais (Eri et Homb; 1947). En Amérique du Nord, (Connecticut), on a fait des observations du même ordre chcz des vaches de races diverses. Quelle que soit la race, la production la plus faible correspond aux naissances de Juin ou Juillet, la plus forte à celles de Janvier-Février, les différences variant entre 13 et $19 \%$ (Frick, Mann et Johnson, 1947).

\section{III. - LE LAIT DES VACHES DE DIVERSES RÉGIONS CHAUDES}

\section{AFRIOUE}

Afrique du Nord. - Le climat sub-tropical de l'Afrique du Nord française fait que, en matière de production laitière, les races européennes ou leurs croisements ont peu à peu supplanté, du moins pour le ravitaillement des centres, les vaches indigènes. C'est particulièrement vrai en Algérie et au Maroc.

Chez les vaches indigènes, les moyennes, dans la composition du lait, se rapprochant de celles d'Europe; cependant, la teneur en matière grasse et matières albuminoïdes est plus forte chez les vaches indigènes que chez les vaches de raccs européennes, le rendement étant par contre, bien moins important. Les chiffres cités par Lamire, Girard, Saillard (1949) montrent combien la production peut être augmentée par le croisement hollandais ou tarentais, et aussi que la teneur en matière grasse diminue comme augmente' le rendement, observation que nous retrouverons chaque fois que sont examinés les résultats du croisement d'une race européenne avec une race tropicale.

Des vaches hollandaises importées au Maroc, fournissent une moyenne de 4.500 litres, avec uno teneur moyenne, en matière grasse, de $3,4 \%$; de leur croisement avec la race marocaine, s'est formée depuis longtemps une race dite noire-pie de Meknès, dont les vaches donnent en moyenne 6 à 7 litres par jour d'un lait très riche en matière' grásse. D'autre part, le métissage avec la tarentaise donne un produit qui fournit 5 à 6 litres par jour avec une teneur de $4,4 \%$ en matière grasse.

En Algérie, de deux sous-races indigènes, la race Guelma-Cheurfa de Constantine et la race de la Chaouia, originaire du Maroc, la première est la meilleure laitière, donnant 7 litres par jour (Cacciaguerra, 1942).

Égypte Soudan égyptien. - En Égypte, où $70 \%$ du lait est fourni par des bufflesses, la production annuelle d'une bufflesse varie entre 4.000 et 5.600 livres, avec une teneur moyenne de 70 à 80 grammes de matière grasse, alors que les vaches donnent de 5.500 à 7.700 livres de lait dont là teneur en beurre oscille entre 35 et 37 grammes.

La composition moyenne, chez les vaches, établie de 1937 à 1944; est de 4,87\% de matière grasse (extrêmes 2,9 et 25 ) et $9,11 \%$ de matière sèche non grasse (extrêmes 7,06 et 10,57 ) (Ghoneim et collaborateurs, 1947).

Les vaches du Soudan égyptien sont meilleures laitières que les vaches zébus de l'Est africain; la sélection et les soins permettent une amélioration rapide de la" production; un même groupe de vaches 
qui donnaient en 19423.874 livres de lait en donnaient en 1944, 4.929 livres du seul fait d'une meilleure éducation des bouviers; $37 \%$ de ces vaches, au cours de quatre années, ont donné plus de 300 gallons de lait ( 1 gall $=4$ l. 54), $20 \%$ plus de 400 gallons et $12 \%$ plus de 500 gallons.

Les vaches du Nord du Soudan égyptien qui paraissent appartenir au type zébu short horn (Royns, 1947) donnent en moyenne 1.954 livres de lait annuellement. Si on les nourrit convenablement, on voit la production monter à 3.874 livres, puis à 4.420. Le record est de 10.272 livres en une année, avec un maximum de 52 livres par jour et une teneur en matière grasse de $4,35 \%$.

Dans le Sud-Ouest du Soudan, on a entretenu des vaches provenant de régions plus au Nord, et non sélectionnées; la moyenne journalière, au cours d'une lactation, est de 61.5 dans certains lots, de 4 l. 2 dans d'autres, la moyenne pour 54 lactations étant de 5 1. 2 par jour (Stamforth, 1948).

Afrique orientale. - Au Kenya, les teneurs moyennes en matière grasse sont de 55 à 58 grammes chez les vaches indigènes. Dans une ferme européenne , où ces vaches étaient sélectionnées, l'une d'elles donnait 2.295 litres en 278 jours avec $5,5 \%$ de beurre, une autre 1.528 litres en 284 jours, avec $5,9 \%$; dans l'ensemble, ces vaches avaient une production double de leurs congénères élevées dans les conditions de l'élevage indigène (Daubney, 1938). Dans les mêmes conditions d'alimentation et d'entretien, Cameron (1945) voit la production passer de 5 l. I en 1933 à 7 l. 9 en 1942, et la durée de la lactation de 75 à 225 jours, la teneur moyenne en matière grasse étant de $5,6 \%$.

La teneur du lait des vaches du Kenya, en matière sèche non grasse, baisse au-dessous du minimum considéré comme légal $(8,5 \%)$ pendant la saison sèche, alors que le pâturage est sec, et qu'on distribue abondamment du concentré; cette baisse est beaucoup moins fréquente en saison des pluies, alors que le paturage est de bonne qualité. C'est le lactose qui baisse. La baisse est plus marquee chez les vaches de races européennes (Friese, Ayshire) que chez les vaches indigènes (Purchase et Reverberi, 1946).

Au Tanganyika, les cinq meilleures vaches de race Ayrshire, à Mpwapwa, ont fourni pendant 300 à 384 jours 5.010 à 7.251 livres de lait avec un maximum de $5,6 \%$ de matièrc grasse, alors que les cinq meilleures vaches zébus donnaient en 283 à 310 jours 3.009 à 3.681 livres de lait, la teneur maximum en matière grasse étant la même. La moyenne journalière est de 15,7 à 18,8 pour les Ayrshire, et de 9,8 à 11,8 pour, les zébus.

A la station centrale d'élevage de Mpwapwa, on poursuit le croisement des femelles zébus avec des taureaux de races Sindhi et Sahiwal, de l'Inde. On cherche à obtenir un type donnant 300 gallons ( 1 gall $=41$. 54) de lait de bonne qualité en 300 jours de lactation, et adapté aux conditions locales avec le minimum de complément alimentaire. En croisant les vaches de race Ayrshire avec les taureaux indiens, on obtient un rendement de 300 à 600 gallons.

En Uganda (station de Lerere), sur 45 vaches de race locale, 10 ont fourni une production dépassant 150 gallons, 14 entre 150 et 100 gallons, 21 au-dessous de 100 gallons. On a pu porter la production laitière de vaches indigènes non sélectionnées à 12-15 livres par jour simplement en leur fournissant une ration bien équilibrée et abondante (Kerkham et collaborateurs, 1947).

En Afrique orientale italienne, où le zébu à cornes courtes est la race la plus commune, la production est de 200 à 500 kilos pour une lactation de 4 à 8 mors.

A Djibouti, Prunier citait en 1939 le cas d'une étable où 40 vaches importées du Yémen, où elles ne donnent que 2 litres de lait environ en pleine lactation, portaient leur production à 8 litres grâce à un entretien soigné et une bonne alimentation.

A Zanzibar (ferme expérimentale de Kizimbani) la proportion de vaches indigènes dont le rendement en lait dépasse 2.000 livres est de $8.5 \%$; entre 1.500 et 2.000 livres, $17 \%$; entre 500 et 1.500 livres, $25,6 \%$ et au-dessous de 500 livres, $48,9 \%$.

Afrique du Sud. - En Afrique du Sud, la production, bien qu'assez hétérogène en raison de l'introduction de diverses races, se rapproche de celle d'Europe beaucoup plus que de celle des autres régions d'Afrique.

On a observé, au cours des dernières années, que, par suite du remplacement progressif du bétail laitier local par des races européennes, la teneur des laits de mélange en matière sèche diminue et, dans la moitié des cas, est inférieure au táux standard de $8,5 \%$, la moyenne pour la matiere grasse etant de $3,59 \%$.

En Rhodésie, les rendements suivants ont été observés dans 9 troupeaux contrôlés officiellement et 145 troupeaux contrôlés semi-officiellement, la majorité des vaches appartenant à la race Friesland. avec d'autres races: Jersey, Guernesey, Red poll, Shorthorn, Ayrshire. Dans l'ensemble, la durée de la lactation est de 200 à 300 jours. Pour les troupeaux officiels, pour 124 lactations, la moyenne est de 7.475 livres de lait avec $3,69 \%$ de matière grasse et une duree moyenne de 289 jours. Dans les troupeaux semi-officiels, pour 4.574 lactations la moyenne est de 5.762 livres, la teneur en beurre de $3,7 \%$ et la durée de 277 jours. 


\begin{tabular}{|c|c|c|c|}
\hline$" .$. & RENDEMENT JOURNALIER & DUREE LACTATION & RENDEMENT ANNUEL \\
\hline 10 Taurins : & Jitres & mois & litres \\
\hline Race N'dama $\ldots \ldots \ldots \ldots \ldots \ldots$ & $1-2$ & $5-6$ & $200-350$ \\
\hline Sous-race Bambara ............. & $1-2$ & $7-8$ & $250-300$ \\
\hline Sous-race Borgou (Dahomey) ... & $1,5-2,5$ & $6-7$ & $300-350$ \\
\hline Race des Lagunes & $1-1,5$ & $5-6$ & $200-300$ \\
\hline Race du Tchad. ................. & $2-4$ & $6-7$ & $600-700$ \\
\hline 20 Zébus : & & & 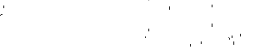 \\
\hline Peul Sénégalais & 2 & $5-6$ & $300-400$ \\
\hline Soudanais & $2-3$ & $5-6$ & $350-450$ \\
\hline Peul Nigerien .. & $2-3$ & $5-6$ & $350-450$ \\
\hline Maure..., . . . . & $2-4$ & 6 & $500-600$ \\
\hline Touareg ...... & $2-4$ & $5-6$ & $350-400$ \\
\hline Azaouak (Niger). . & $2-5$ & $6-8$ & $500-600$ \\
\hline Bororodji (Niger) ....... & $1,5-3$ & 6 & $350-400$ \\
\hline
\end{tabular}

Afrique occidentąle française. - Le rendement en lait des diverses races de l'Afrique occidentale française se chiffre en moyenne de la façon suivante (Mornet, 1948). (Voir tableau ci-dessus).

L'influencc du sang zébu se fait sentir dans le croisement avec les bovins sans bosse du Soudan : alors que les taurines du Sud ne donnent guere que 1 à 2 litres par jour, les métis donnent 2 a 3 litres pendant une période de 5 à 6 mois, et 5 à 6 litres quand les éleveurs indigènes leur donnent des suppléments.

La vache maure, en brousse, fournit facilement 5 litres de lait par. jour, et plus en bonne saison. Elle peut donner 8 litres et, 'suralimentée avec de la paille d'arachides, du mil et du son, jusqu'à 10 litres el exceptiomellement 12 (Prigent et collaborateurs 1942).

En saison sèche, la teneur en beurre peut varier entre 27 , et 81 grammes par litre, avec une moyenne de 50 grammes. En saison des pluies, la moyenne est de 58. grammes avec des extrêmes de 33 et 81 . Quant au lactose, il varie beaucoup moins : en saison sèche, 44 à 54 grammes avec moyenne' 50; en saison des pluies : 45,6 et 62,3 avec moyenne 58,38 .

$L^{\prime}$ extrait sec, en saison sèche, varie entre 137 et 185 grammes, avec une moyenine de 162 grammes. Peu après la mise bas, on peut trouver 180 et même 190 grammes d'extrait sec, 75 à 85 grammes dé matière grasse et 60 à 80 grammes de matières albuminoïdes. A la fin de la lactation', on peut noter des chiffres encore supérieurs.

Au Soudan Français (Curasson, 1933) des variations extrêmement marquées sont observées : extrait sec, de 105 à 194, moyenne 149; matière grasse de 15 à 101, moyenne 50; matières albuminoïdes de 32 à 124, moyenne 52; lactose, moyenne 47. Des vaches indigènes entretenues dans une ferme de la même région ont donné lieu en 1940 aux observations suivantes : Densité de 1026,5 à 1035; acidité Dornic 20 à 26; matière grasse 42 gr. 5 à 92 gr. 8 (Doutressoulle, 1947).

Au Sénégal (Dufour, 1932) 'la moyenne de nombreuses analyses est de plus de 55 pour la matière. grasse, 40 pour la caséine, le lactose se maintenant autour de 50. 'La teneur en anhydride phosphorique est constante $(1,5)$.

Dans l'ensemble, les moyennes sont les suivantes:

\begin{tabular}{|c|c|c|c|}
\hline \multirow[b]{2}{*}{ Densité. .............. } & \multirow{2}{*}{$\begin{array}{l}\text { Moyennes } \\
1.030,1\end{array}$} & \multicolumn{2}{|c|}{ Extrênes } \\
\hline & & 1.037 & $1.024^{\circ}$ \\
\hline ec non dégraissé. & 149,7 & 194 & 105 \\
\hline & 50,4 & 101. & 15 \\
\hline & $.52,1$ & 124 & 32 \\
\hline ose. & 49.5 & 54 & 4 \\
\hline
\end{tabular}

Le lait de vaches indigènes entretenues à la ferme de Sotuba (Soudan français) a donné lieu, en 1940, aux observations suivantes (Doutressou).

\begin{tabular}{|c|c|c|}
\hline & Maximurn & Mininum \\
\hline Der & 1.035 & $1.026,5$ \\
\hline $\begin{array}{l}\text { Acidité Dornic } . . . . . \\
(2 \text { heures après traite })\end{array}$ & $26^{\circ}$ & $20^{\circ}$ \\
\hline sses. & $92 \mathrm{gr}$ & 42 \\
\hline
\end{tabular}

En ce qui concerne les matières minérales, il y a déficit en acide phosphorique, chlore, sodium, 
magnésie. Le taux de potasse est, par contre, supérieur à celui d'Europe. La chaux augmente considérablement en saison humide par rapport à la saison sèche.

Afrique occidentale anglaise. - En Sierra-Leone, duction laitière des vaches N'dama est la suivante dans deux stations différentes : dans l'une, pour une période de lactation de 194 à 258 jours, la production annuelle est de 295 à 1.510 livres, le maximum journalier étant de 13 livres 1/4; dans l'autre station, pour 237 jours de moyenne, la production annuelle moyenne fut de 1.071 livres, le pourcentage de matière grasse étant de $7 \%$.

En Gold Coast, les vaches indigènes de race Shorthorn donnent en moyenne 140 à 180 litres de lait. en une période de lactation de 100 à 180 jours. Exceptionnellement, on observe une production de 675 litres; des vaches zébus importées de Nigéria ne donnent, à la côte, que 3 litres en moyenne. A la ferme de Pong-Tamalé, on a tenté la création d'un bétail laitier à partir du zébu (White Fulani) ou du métis Sanga, qui peuvent donner régulièrement un minimum de 21 . 1/2 par jour. Les Shorthon n'arrivent pas à ce taux.

La teneur moyenne en matière grasse était, en 1936-37, la suivante : vaches N'dama : 4,1\%; Métis Zébu-Shorthorn 3,2 à $3,8 \%$ : Shorthorn, $3 \%$.

Comme en d'autres régions, l'influence de l'alimentation se fait sentir et est illustrée par l'observation suivante : dans une ferme, l'alimentation et le bon entretien permettent à la petite race locale du Nord de donner 1.201 livres de lait en 301 jours de lactation.

En Gambie, la production des vaches de race locale est faible; un chiffre peu ordinaire est 4 l. 5; la teneur en matiere grasse est de $6 \%$ et plus.

En Nigeria, en 1946, le rapport moyen des vaches de race Peul blanche (White Fulani) était de 2.336 livres, soit 61.18 par jour entre deux vêlages: celui des vaches de race Gudali, de 2.727 livres, soit 6 l. 98 par jour entre deux vêlages. L'influence du mâle a été observée : le meilleur taureau peul fournit des descendantes dont la production atteint 3:014 livres; celles du meilleur taureau Gudali, 3.652.

Afrique équatoriale. - Les races indigènes de l'Afrique équatoriale se rapprochent, quant aux aptitudes laitières, de celles de l'Afrique occidentale.

Dans sa zone d'élevage, le bœuf "Kouri », bœuf du Lac Tchad, est considéré comme de bons caractères laitiers, le rendement quotidien d'une borne vache pouvant atteindre 8 litres, et la lactation durant 6 à 7 mois, exceptionnellement 10 mois.

Au Congo Belge (Pigneur, 1930) le lait des vaches indigènes est considérablement plus riche cn matière grasse et matieres albuminoides, alors que la teneur en lactose est sensiblement la même (Els, 1931) que celle du lait des vaches d'Europe.

Au Ruanda Urandi, les observations comparées des rendements laitiers des, vaches sans cornes et des vaches à cornes donnent les résultats suivants : bétail à cornes, en 381 jours, 829 litres avec $5 \%$ de matiere grasse; bétail sans cornes : en 384 jours, 756 litrcs avec $6.1 \%$ de matière grasse.

Au Cameroun, les femelles de type zébu ceul donnent habituellement 2 litres $1 / 2$ à 3 litres de lait après vêlage, et 2 litres à peine apres 2 ou 3 mois de lactation. Par contre chez un autre type de zébu dit Akou la production journalière peut être de 5 à 6 litres.

Madagascar. - Chez la vache zébu de Madagascar, on ne récolte guère que 150 à 250 litres au cours d'une lactation qui, en général, n'excède pas 6 mois. Le pourcontage des matières grasses est élevé, et dépasse souvent $5 \%$.

Chez les métisses normandes, aux mains de Malgaches, qui ne leur donnent ni concentré ni mélange minéral, ni foin, ni ensilage, la production varie de 400 à 800 litres par lactation, la teneur en matières grasses varient de 4 à $4,5 \%$. Par contre, chez les éleveurs européens ou les éleveurs indigènes qui les soignent mieux, une production moyenne de 1.500 litres en 300 jours n'est pas rare; à la laiterie de Tamatave, 30 vaches métisses pesant de 350 à 500 kilos ont donné en un an 57.000 litros de lait, soit environ 1.900 litres par tête. Les métisses normandes de $2^{\mathrm{e}}, 3^{\mathrm{e}}, 4^{\mathrm{e}}$ génération dépassent 2.500 litres en 300 jours. Une vache normande née à Madagascar de père et mère eux-mêmes nés dans l'Ile a donné 4.200 litres de lait en 330 jours (Guillermo, 1949).

\section{ASIE}

Proche et Moyen Orient. - En Palestine, les vaches de race locale donnent 500 à 600 litres de lait par an; on double ce chiffre par la sélection et l'entretien. Les vaches importées de Syrie et du Liban sont meilleures laitières : 1.200 et 2.000 litres respectivement. Les vaches de Friese importées ne résistent pas au climat, mais leurs croisements avec la race syrienne donnent de 3.000 à 3.200 litres. D'autres observations relèvent que la teneur en matiere grasse du lait des vaches locales varie entre 4 et $5 \%$. la production annuelle allant de 1.600 à 1.800 kilos. Chez les animaux provenant du croisement avec un taureau frison, la production s'éleve à 3.800 à 4.000 kilos, avec une teneur en matière grasse de $3,75 \%$ \%

La race qui, dans les zones agricoles riches de Syrie, est bonne laitière, ressemble un peu à la vache de Jersey. Elle donne 3.000 à 3.500 kilos de 
lait en une lactation de 195 à 300 jours (moyenne 245). Certaines vaches, au maximum de la láctation, peuvent produire 23 kilos par jour (Ferrando, 1941).

En Irak, où la plus grande partie du lait est fournie par des bufflesses, les bonnes bêtes donnent au maximum 3 gallons ( $=13$ l. 5 env.) par jour. Parmi les bovins, il existe un type zébu, le type Jenubi, supérieur aux autres, dont les vaches peuvent donner 15 litres.

Inde. - Bien que l'Inde possède 215 millions de bovins, soit environ le tiers de la population bovine du globe, la production laitière est très basse, la production moyenne annuelle, par vache, n'etant que de 525 livres environ. Sur ces 215 millions, il y a 50 millions de buffles, qui fournissent la moitié de la production, la moyenne étant, pour la bufflesse, de 1.270 livres (Lazarus, 1946).

La différence ne tient pas seulement à la quantité, mais aussi à la composition. On admet en général que la teneur du lait de vache en matière grasse est le double de celle d.'Europe "et celle du lait de bufflesse le triple, ce qui paraît exagéré. La réglementation considère comme pur le lait de bufflesse qui a une densité minima de 1,028 à $15^{\circ}, 90$ grammes de matière sèche non graisseuse et 60 grammes de matière grasse; un tel lait, dilué, pourrait passer pour du lait de vache, si ce n'était l'abaissement du taux du lactose.

Il y a d'autres differences entre le lait de vache et le lait de bufflesse. C'est ainsi que le lait de vache de l'Inde a une teneur en phosphatase similaire de celle du lait de brebis, alors que celle du lait de bufflesse est similaire de celle du lait de chèvre (Kannan et Basu, 1948). D'autre part, les laits de l'Inde diffèrent de ceux d'Europe quant à la répartition des acides gras, différences qui paraissent d'ordire climatique (Banerjee, 1946).

La production est très variable, en raison de la diversité des races locales et des croisements d'importation.

Dans l'Etat de Mysore les vaches de race locale (Amrit Mahal) donnent, dans une ferme, une moyenne de 91.27 de lait, en dehors de ce que prend le veau, quand on ajoute, à la ration ordinaire, des concentrés; celles qui ne sont pas ainsi traitées ne donnent que 6, 1. 9.

Dans une ferme militaire où on poursuit l'amélioration de la race locale de Tharparkar, la sélection, les méthodes d'entretien et d'alimentation ont permis de faire passer la production, par jour de lactation. de 8 l. 7 en 1930 à 201.5 en 1945.

Dans la zone montagneuse des Provinces Unies, la petite race locale donne très peu de lait en une période de lactation qui n'excède pas 150 jours. Mais 'alimentation, le mode d'entretien permettent une amélioration considérable : une vache bien entretenue à l'Institut de Mukteswar a donné, au cours de quatre lactations. successivement 753, 662, 804 et 927 livres de lạit, la durée de la lactation étant de 241, 153, 196 et 273 jours.

L'introduction, dans le sang zébu, de sang euro' péen par le taureau, amène une rapide amélioration. De forts rendements sont obtenus par les HolsteinFriese croisés avec les vaches de race Sahawal Ie croisement Jersey-Sindh, plus petit, donne un peu moins d'un lait plus riche. A l'Institut agricole d'Allahabad; on a d'abord remarqué que le taureau Jersey avec la vache Sindh donne de bons résultats quand on s'en tient au demi-sang ou au quart de sang, la production moyenne étant, par jour, de 1.428 livres pour i84 vaches; au premier croisement, on obtient moirs de 2.000 livres par lactation, puis le rendement augmente chez les descendants, de deux à trois fois. La production se fixe chez les vaches qui ont environ 1/16 de sang Jersey.

Aux Indes, les races laitières européennes ou les métis proches du sang ne donnent pas satisfaclion. Dans une ferme. modèle le rendement moyen des métis (pour la plupart Holstein) était le suivant pour une lactation en 1941 .
$1 / 8$ sang................. $2.199 \mathrm{~kg} .54$
$1 / 4$ sang................ $2.114 \mathrm{~kg} .09$
$1 / 2$ sang.................. $3.171 \mathrm{~kg} .36$
$5 / 8$ sang................ $3.175 \mathrm{~kg} .06$
$3 / 4$ sang................ $3.029 . \mathrm{kg} .09$
$7 / 8$ sang................. $2.809 \mathrm{~kg} .09$

La production baisse donc, à partir de $5 / 8$ de sang, à mesure qu'augmente la proportion de sang européen.

Dàns les États Malais, la durée de la lactation chez les bufflesses est de cinq à six mois pendant lesquels on obtient par bufflesse en moyenne 540 lilres, sans compter ce qui est utilisé par le veau.

A. Ceylan, il y a quatre types de bétail : la race indigène (Sinhale), des races indiennes (Sindh et Sahiwal), des races européennes importées; et des métis. La capacité de production de ces divers types est la suivante, pour une lactation (Wright, 1945).

\begin{tabular}{|c|c|}
\hline $\begin{array}{l}\text { Race indigène (Sinhala). .... } \\
\text { Races indiennes }\end{array}$ & $\begin{array}{r}363 \mathrm{~kg} \cdot 6 \\
1.136 \mathrm{~kg} \cdot 36\end{array}$ \\
\hline Métis ......... & $1.477 \mathrm{~kg}$ \\
\hline Races européennes & $1.704 \mathrm{~kg}$ \\
\hline
\end{tabular}

On peut considérer que, dans l'ensemble; les vaches de Ceylan donnent I litre à I litre 1/2,' parfois 3 litres; les vaches zébus de race Sinhala, dans les conditions ordinaires d'élevage, donnent 180 à 270 litres et les bufflesses de 270 à 360 litres. Le meilleur élément améliorateur parait la race Red Sindh dont les métis fournissent un peu moins de 
lait que les métis européens, mais sont plus résistants.

Aux Indes occidentales, où les bovins représentent un croisement de la race locale avcc le zćbu, les caractères de ce demier dominant, la lactation dure plus de 100 jours, parfois 150; la production annuelle varie de 225 à 400 litres ou de 575 à 675 pour les annimaux mieux entretenus, la teneur en matière grasse étant de 4,6 à $4,8^{\circ} \%$ et plus. Le croisement avec les taureaux demi-sang ou trois-quarts sang Holstein permet de porter le rendement à 7 ou 9 litres par jour et de prolonger la durée de lactation jusqu'à 200 à 240 jours. Les bêtes de race Holstein pure, ou proches du sang sont inférieures (Miller, 1948).

Les bufflesses des Philippines pourraient fournir une sécrétion lactée pendant 12 ans et même 18 ans, les bufflesses hindoues jusqu'à 17 ans, les moyennes hebdomadaires en litres pouvant etre de 2,412, $18,283,6,390$ et 15,120. Les bufflesses de Delhi, réputées, pourraient fournir 18 à 24 litres par jour.

La composition moyenne du lait de bufflesse (en Europe) cat la suivanto :

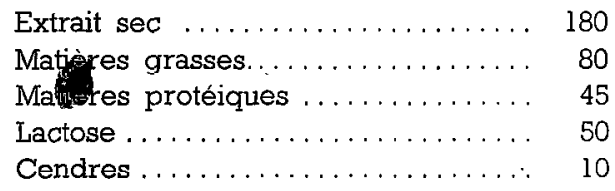

Au cours d'une période de lactation, les vaches chinoises donnent en moyenne 300 à 400 livres de lait, mais la production a atteint 2.935 livres dans un cas individuel. La teneur moyenne en matière grasse est de 6,6 \% (Lee R.-Y., 1946).

Indochine. - La production journalière des vaches indigènes oscille entre 1 litre et. 1 litre $1 / 2$. atteignant exceptionnellement 2 litres. Cependant, au Tonkin, de petites vaches à robe brune et cornes courtes, bien nourries, peuvent donner 2 litres $1 / 2$ et 3 litres et, suralimentées, 5 litres. Dans l'ensemble, le rendement moyen est de 200 à 350 litros par lactation (G. Guy, 1945).

Diverses races européennes ont été introduites : bretonne, normande, hollandaise, tarentaise, bordelaise; ces tentatives ont, dans l'ensemble, échoué, en raison des épizooties notamment. De même pour des vaches en provenance d'Australie.

Le croisement qui prédomine est le croisement avec la race de l'Inde dite Sindh, qui a essaimé dans de nombreuses régions tropicales. La durée de la lactation est plus longue chez ces métis. Ainsi, cette durée se présente de façon suivante : 5 a 6 mois chez les vaches annamites; 7 à 8 mois chez les 1 , 4 Sindh; 8 à 9 mois chez les $1 / 2$ Sindh; 9 à 10 mois chez les 3/4 Sindh; jusqu'à 12 mois chez les métisses Sindhfrançais (Jauffret et Autret, 1947-48).
Voici, à titre de comparaison, les rendements de diverses variétés de vaches chez des producteurs de lait annamites :

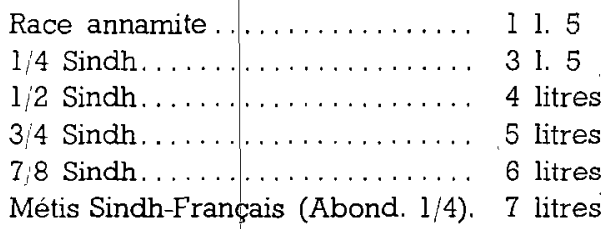

Dans la région de Dankia (Evanno) une vache bordelaise, en cours de lactation, a donné pendant les 118 premiers jours, 2.300 litres de lait dont le taux en matières yrasses élait en muyenne de 56,38 . Une autre bordelaise a donné une moyenne de 15 litres pendant 12 mois. Au dixième mois, le taux beurrier était de 73,8 , la teneur en lactose de 40,2 , en caséine, de 44,5, la densité de 1.036. Une troisième, en 389 jours de lactation (non terminée) a fourni 3.452 litres de lait de densité 1.035, la teneur en lactose étant de 41,15 , en caséine de 37,8 et le taux beurrier de 85,4.

Dans la même région, une bretonne pie-noire a donné quotidiennement 8 litres, la composition du lait d'un jour était : beurre 85,4; lactose 40,3; caséine 37; densité 1.033 .

La bufflesse indochinoise qui n'est pas exploitée pour le lait, ne donne, d'après Schein, qu'environ 1 litre à 1 litre $1 / 2$ par jour.

En ce qui concerne la composition, il est évident que la variété dans les croisements s'oppose à l'uniformité. Dans l'ensemble, les principales caractéristiques du lait des vaches autochtones, sont, d'après divers auteurs, les suivantes :

\begin{tabular}{|c|c|c|}
\hline & $\begin{array}{l}\text { Vaches } \\
\text { indigènes }\end{array}$ & $\begin{array}{c}\text { Vaches } \\
\text { d'Europe }\end{array}$ \\
\hline Densité. & $1.025-1.031$ & 1.032 \\
\hline Matières grasses & $60-65$ & 35 \\
\hline Lactose. . & 45 & 48 \\
\hline Caséine & $45-50$ & 29 \\
\hline Extrait sec. & $165-168$ & 127 \\
\hline Extrait sec dégrai & 102,5 & 92 \\
\hline Cendres & $7-7,3$ & 7,5 \\
\hline
\end{tabular}

D'après Bloch et Ch. Richet (1933) le taux de lactose atteint exceptionnellement 50 grammes; la caséine dépasse souvent 50 grammes et les cendres se tiennent entre 7 et 8 grammes. A Hanoï, Lozach a obtenu les résultats suivants, tirés des analyses d'une année : beurre : 67 (de 43,9 à 91); caséine : 50 (de 39.5 à 59,9 ); lactose : 45 (de 41 à 48,7 ); cendres : 7,4 (de 5,5 à 9,9 ).

Les laits du Tonkin, d'après Jauffret et Autret (1947), ont un extrait sec élevé dû surtout à leur richesse en beurre et caséine; ils sont un peu moins riches en lactose; leur minéralisation est caractérisée 
par une haute teneur en phosphate et en calcium et une faible teneur en chlorure de sodium. La teneur en beurre, plus forte en saison sèche, varie en raison inverse de la production. Dans les laits de mélange, la moyenne de matière grasse est de 49 gr. $5(43,8$ a 53,9 ) pour la traite du matin et de 55 gr. 6 (de 51,9 à 69,1$)$ pour la traite du soir. Dans des laits individuels, on peut trouver $67 \mathrm{gr} .10$ (traite du matin) et 94 gr. 50 (traite du soir). La teneur moyenne en caséine réelle, dans les laits de mélange, est de 44 grammes (sans différence sensible entre les traites du matin et du soir). La moyenne du lactose est de 44 grammes, celle des cendres de 8 grammes; il y a de 2 gr, 35 à 3 gri 10 de phosphate et de I gr. 90 à $2 \mathrm{gr}$. 20 de calcium, chiffres supérieurs à ceux des laits de France.

\section{AMÉRIQUE}

Les essais les plus importants faits en Amérique du Nord en zone chaude sont ceux qui ont eu lieu dans le Sud du. Texas, où l'introduction du zébu de l'Inde (Brahman) réalisée depuis plus de 100 ans. a permis l'obtention de la " race " dite de Santa Gertrudis, maintenant répandue dans les Etats du Sud; c'est avec des Shorthorn que fut réalisé le croisement; le rendement laitier a été amélioré, mais c'est surtout vers la production de la viande qu'on s'oriente.

Les qualités laitières de la race Sindh de l'Inde, ont été mises à profit pour obtenir en Louisiane un bétail à bonnes qualités laitières et plus résistant que la race de Jersey, objet du croisement.

Le zébu a été utilisé également en Amérique du Sud.

$\mathrm{Au}$ Brésil, en zone chaude, les vaches de race Holstein voient leur production baisser de $56 \%$ alors que, dans les mêmes conditions des croisements entre vaches européennes et Brahman avaient un meilleur comportement que les Brahman et moins bon que les parents européens (Villegas, 1939).

A la Jamaigque, Howe (1946) compare l'influence du sang zébu sur trois races introduites en région tropicale : Jorsey, Gucrnescy, Holstein (notons que, le terme Holstein ou Friesian Holstein est utilisé en langue anglaise pour désigner la hollandaise pienoire). Alors que les races pures donnent 4.020 livres de lait (Jersey), 3.545 (Guernesey), 5.506 (Holstein), les chiffres, pour les demi-sang sont respectivement $4.520,3.993$ et 5.506 livrès. La teneur en matière grasse est également influencée par le sang zébu : les demi-sang Jersey donnent un chiffre de 5,89\% soit $1 \%$ de plus que les vaches pures; les demisang Guernesey, $5,27 \%$ (pures : 0,42 de moins); les derni-sang Holstein, 0,42 \% (7/8 de sang : $1 \%$ de moins).

\section{IV. - MODES D'ACTION DES FACTEURS CLIMATIQUES}

Au cours de cette étude, nous avons vu que certaines observations ont pu établir la part réelle qui revient, dans l'action du climat, à certains facteurs de celui-ci, comme la chaleur et l'humidité. Par. contre, bien souvent on confond l'action propre du climat et celle des facteurs secondaires.

C'est évidemment par action sur le métabolisme qu'intervient le climat dans la sécrétion laitière. Aussi était-il intéressant d'étudier comment réagit la vache laitière aux augmentations de température; c'est ce qu'ont fait Regan et Richardson (1938). Ainsi que nous l'avons vu', le nombre des mouvenients respiratoires augmente régulièrement avec là température; 'à la température de $26^{\circ} 6-29^{\circ} 2$, variable avec la race, on atteint un point thermique où il n'est plus possible aux animaux de maintenir nettement la régulation de la température. Au delà de ce point, il y a inappétence, la production du lait diminue et cé lait est modifié; il renferme moins de caséine et de résidu sec autre que la matière grasse, alors que la matière grasse augmente. C'est un fait observé dans toutes les régions chaudes: Le $\mathrm{pH}$ du lait est plus élevé, le point de congélation abaissé et la coagulation par la présure se fait plus tardivement. La matière grasse est plus pauvre en acides volatils et plus riche en constituants non saturés. D'après ces deux auteurs, le principal moyen de la vache, pour combattre la chaleur, est le système respiratoire. La respiration varie directement avec la température ambiante, la peau a un grand pouvoir d'isolement. La vache peut donc s'adapter facilement au, froid, mais elle est mal préparée pour résister au chaud.

Certaines remarques tendenl à fixer les limites d'intervention du climat lui-même; ainsi les analyses d'Edwards (1938) portant sur le lait de vaches jerseyaises inscrites au "Livre de Mérite 》 dans les Etats du Maine et de Géorgie; vaches ayant une alimentation de haute valeur, montrent que le climat a un effet direct : une diminution au cours de l'hiver pouvait être attribuée au fait que les étables étaient chauffées.

Dans les observations de Régan et Richardson que nous avons citées, quand la température monte jusqu'à $40^{\circ}$, la production laitiere, dans les mêmes conditions d'alimentation, tombe à 17 litres par jour alors qu'elle était de 29 litres à $10^{\circ}$. Les remarques de Rhoad (1935), au Brésil, montrent par ailleurs que les vaches de races européennes introduites dans les régions tropicales et recevant une ration équilibrée ne fournissent que $56 \%$ de leur capacité normale. Le fait que les vaches de races européennes produisent plus quand il fait froid est illustré par'les observations de Villegar que nous avons relatées 
plus haut et dans lesquelles les vaches placées dans une étable climatisée à $20^{\circ}$ donnaient 26 litres de lait par jour, alors qu'un groupe similaire, dans une étable ouverte, ventilée, exposée à la température tropicale, ne donnait que 9 litres.

L'effet de la chaleur est donc démontré; mais ce n'est pas le seul facteur : la perspiration cutanée, les autres conditions météorologiques, notamment l'humidité, la gymnastique fonctionnelle et surtout l'alimentation, interviennent. Cameiro (1939) pense que quand le bétail laitier est soigneusement alimenté, il n'y a pas de baisse marquée de la production et que c'est sur la nutrition qu'intervient le climat; Hammond (1931) pour la Jamaique, Rhoad (1935) pour le Brésil ont montré que la longue période de sécheresse caractéristique de nombreuses régions tropicales réduit considérablement la production laitière en diminuant la capacité des pâturages et la valeur nutritive des herbes. C'est au cours de cette période de disette qu'on voit chez les vaches indigènes qui n'ont alors qu'une alimentation déficiente se produire la baisse marquée dans la quantité et aussi les variations qualitatives.

L'action de la chaleur sur le métabolisme retentit sur l'appétit; le mëtabolisme est d'autant plus affecté que l'animal est un bon transformateur de nourriture; aussi, les bêtes grosses mangeuses, comme les laitières, mangent moins, sécrètent moins de lait. C'est pourquoi, font remarquer Scholtz et Badenhorst (1940), à la Jamaïque, au Congo Belge, on les croise avec des zébus, et pourquoi aussi la Jerseyaise, petite et mangeant peu, est la seule race qui réussisse dans les régions chaudes et sèches de l'Amérique.

Les climats chauds interviennent, d'autre part, indirectement sur l'alimentation en diminuant l'activité de lá vache au pâturage. Cette diminution de la quantité d'aliments nécessaires est vérifiée par des expériences des auteurs sud-africains qui, plaçant sur les mêmes pâturages, au soleil, des animaux d'origines diverses, observent que les bovins indigènes pâturent plus longtemps; de même, la rumination cesse plus vite au soleil chez les races importées. Il faut remarquer aussi que, lorsqu'il y a du vent, les animaux pâturent plus longtemps avant de se coucher et que, quand il fait chaud, ils pâturent contre le vent alors que, lorsqu'il fait froid, ils pâturent dans le sens du vent.

D'autre part, au-dessus de $26^{\circ} \mathrm{C}$., l'action indirecte de la température s'exerce aussi en diminuant l'utilisation de l'eau et de la nourriture absorbées.

Il est possible aussi que, dans l'abaissement de la production en période chaude, intervienne le système circulatoire, le nombre des pulsations étant diminué, et par suite l'apport d'éléments consti- tutifs du lait par le sang circulant.
D'ailleurs, le sang peut subir dos variations saisonnières qui retentiraient sur la composition du lait. C'est ainsi que, chez des vaches de l'Inde, on note des variations saisonnières marquées en ce qui concerne la teneur du sang en fer, calcium et magnésium, le maximum étant en été. Il y a également des différences dans le volume des globules rouges et la teneur en hémoglobine, avec maximum en hiver (Pal, Momin et Mullick, 1945).

L'influence du climat se fait vraisemblablement sentir aussi par action sur les glandes endocrines génératrices d'hormones. Ce qui est notable, c'est la remarquable labilité du métabolisme basal chez la vache laitière, et l'accroissement marqué causé par la lactation. Les différences entre le bélail de boucherie et le bétail laitier, à ce point de vue, permettent de penser que la production de chaleur varie avec diverses adaptations fonctionnelles. Ces dernières étant causées par des hormones (Ritzmann, 1938).

On sait d'ailleurs le rôle capital de certaines hormones dans la lactation, notamment la prolactine de l'ante-hypophyse; le rôle de la thyroïde est, d'autre part, illustré par l'action des thyro-protéines qui enrichissent le lait en matière grasse. Or, on sait que la chaleur, les ions négatifs de l'atmosphère influent directement ou par le canal du sympathique sur les fonctions de la thyroide, de l'hypophyse. La pituitaire, qui joue un rôle dans la régulation thermique voit son activité accrue; si les fluctuations thermiques sont peu marquées, l'influence est peu augmentée, et une longue période de chaleur uniforme la ralentit. Il est donc possible que dans les régions à température uniformément chaude pendant longtemps, il y ait inactivité de la pituitaire antérieure, inactivité qui pourrait agir sur la sécrétion lactée, comme elle intervient pour diminuer la croissance du squelette et l'activité sexuelle.

\section{CONCLUSIONS}

Les exemples sont nombreux, qui nous ont été offerts au cours de cette étude, de l'importance relative de l'action directe ou indirecte du climat; mais cette action reste limitée. Le milieu agit beaucoup par l'intermédiaire de la flore, et cette action peut être atténuée par l'amélioration de l'alimentation. Si on dispose de fourrages toute l'année, si on supprime les carences d'ordre divers, si on supprime aussi les causes d'infection, il est quantité de tentatives d'acclimatement qui, considérées comme dangereuses, deviennent faciles. Les meilleurs exemples nous viennent des régions tropicales américaines, mais ils existent aussi en diverses régions asiatiques et africaines. Aussi Jardine, considérant l'amélioration du zébu du Kenya par les races européennes importées, en arrive-t-il à conclure 
que, dans les fermes à gérance européenne, on arrive à tous lés degrés de croisement, et que les facteurs essentiels sont la situation au point de vue maladie et au point de vue alimentaire. "Dans les régions saines, le seul obstacle à une amélioration est le facteur alimentaire. Un bétail de qualité supérieure demande des conditions supérieures d'alimentation, d'eau et de pâture. Là, où ces conditions sont remplies, on a établi. des troupeaux qui peuvent se comparer à la fois pour l'apparence et pour les performances aux troupeaux d'Europe. - Dans Jes conditions semi-tropicales qui sont celles des hauts plateaux du Kenya, on n'a pas encore observé jusqu'à présent, sur les fermes, qui s'occupent uniquement de la production du bétail de haule qualité, d'obstacles inhérents' à la continuation du processus d'amélioration d'une façon indéfinie; en outre, il y a actuellement sur la colonie plusieurs troupeaux purs de races européennes dont la progéniture ne présente aucun signe de dégénérescence, mais plutôt le contraire 》.

Des remarques analogues ont été faites au Cameroun, mais là, comme au Kenya, on pourrait invoquer l'action modératrice de l'altitude, facteur qui ne peut compter dans les essais réussis en zone vraiment tropicale, et dont nous avons vu des.exemples. Les observations faites par Ostertag (1937) sur la « laiterie tropicale " ont bien montré que c'est par la lutte contre les épizooties et par l'amélioration de la flore alimentaire qu'on peut réussir la création de troupeaux laitiers.

D'autres fois; 'c'est la faune, parasitaire ou non, qui joue le rôle primordial; il est évident que quantité de régions tropicales sont fermées à l'élevage, נו que l'introduction d'animaux non indigènes y est interdite, uniquement en raison de la présence des hôtes vecteurs des trypanosomiases et des piroplasmoses et non en raison du climat seul. Que disparaissent ces agents, et l'élevage devient facile. Cela explique que, sur les plateaux africains au delà de 1.500 mètres environ, alors que disparaissent les glossines, on a pu croiser ou élever des races européennes; plus bas, reparaissent les mouches et le danger.

Le facteur humain est, lui aussi, trop souvent négligé : conditions sociales, politiques, religieuses, degré d'instruction, de civilisation, moeurs, etc. $\mathrm{Ce}$ sont ces conditions qui guident les modalités de l'action amélioratrice et fixent les règles utilisables; dans deux pays semblables au point de vue climalique ou même dans un seul pays, les possibilités de réussite varient' beaucoup selon que l'élevage est laissé aux mains dẹ l'éleveur indigène peu évolué, ou qu'il est conduit ou pràtiqué par l'éleveur européen. Un colon qui a su discipliner les facteurs secondaires dư climat réussit ce que ne peut tenter. à côté, l'ćlcvcur indigène d'une « réserve ». L'oubli de l'importance de ce facteur humain a fait échouer bien des tentatives et émettre bien des prétentions qui ne pouvaient être suivies de réussite et dont l'échec était attribué faussement au climat. C'est ainsi que, périodiquement; on compare la rapidité avec laquelle l'élevage des zones torrides et sèches de l'Amérique du sud a évolué, à la stagnation relative de l'élevage africain dans des régions semblables; mais on oublie le facteur éleveur.

L'amélioration de l'alimentation ne doit pas seulement avoir effet sur la quantitć de lait produite, mais aussi sur sa composition. $\mathrm{Si}$, chez les vaches sous-alimentées, le taux de matière grasse reste à peu près fixe, la densité et l'extrait sec baissent (Lutz, 1947); mais si la teneur en matièré grasse est inférieure à $3 \%$ de la matière sèche, il en résulte une baisse de la teneur en beurre du lait, baisse qui peut atteindre l'l \%. Par ailleurs, la pauvreté de beaucoup de fourrages tropicaux en phosphore et en calcium est une cause de déficience du lait en quantité, beaucoup qu'en qualité, la teneur en phosphore et calcium de la ration agissant moins sur la composition du lait que sur la quantité sécrétée.

Nous avons, au cours de cette étude, indiqué maints exemples qui montrent que la. sélection et l'alimentation peuvent considérablement améliorer la production laitière de vaches rustiques. Indiquons encore que, dans l'Etat de Mysore, des vaches qui reçoivent 3 livres d'aliment concentré par jour, plus I livre pour 3 livres de, lait, fournissent 9 à 27 livres de lait par jour alors que les vaches de même race, si elles ne reçoivent pas d'aliment concentré, donnent de 6 à 9 livres de lait (Melsaac, 1941). Des remarques du même ordre sont faites chez des vaches d'une autre région de l'Inde, dont la production journalière passe de 8,7 livres en 1930 à 20,5 livres en 1945 par sélection et alimentation (Ogilvie, 1947) et d'autres vaches de l'Etat de Mysore, dont la production journalière passe du simple au double en 10 ans (Sastri, M. Krishna, 1943).

Le mode d'entretien à l'étable doit avoir aussi son influence. Des expériences, faites en Californie, ont montré que chez des vaches maintenues à l'étable, la température corporelle reste constamment à 3803-3804 tant que la température de l'étable ne dépasse pas '2l ; l'augmentation est ensuite progrossive jusqu'à atteindre 4005 quand la température ambiante est de 37-380 (Hornby, 1942).

Des soins hygiéniques peuvent aussi tempérer l'effet' de la chaleur : les douches ont, chez la 'butflesse, une action évidente sur la production du lait, en abaissant la température corporelle. Au cours d'une période chaude (plus de $44^{\circ}$ ) l'usage régulier de la douche, avant la traite, assure une production régulière; si on cesse cette pratique, la production 
laitière devient irrégulière. On asperge avec de l'eau à $25^{\circ}$ environ pendant 5 minutes, 2 heures avant la traite (Sinha et Minet, 1947).

Une part importante doit revenir aussi, dans l'amélioration de la production des races primitives, à la gymnastique fonctionnelle, dont lo mécanisme est maintenant éclairé par le rôle de l'ante-hypophyse.

Une fois commencée, la lactation est entretenue par une excrétion continue de l'hormone antehypophysaire galactogène : alors que l'hypophysectomie suspend la lactation, les extraits antehypophysaires provoquent, chez les vaches au déclin de la lactation, une augmentation du rendement laitier. Par voie réflexe, la succion et la mulsion entretiennent la sécrétion de la prolactine. "Le système nerveux $a$, dans la lactation, un rôle de conducteur d'influx sensitif débutant soit dans l'utérus, soit dans le mamelon et stimulant la sécrétion ante-hypophysaire de prolactine » (Lesbouyries).

Une expérience faite dans une station de l'Institut des recherches agricoles de New Dehli montre bien l'influẹce de la gymnastique fonctionnelle sur des vaches de races rustiques : un lot de vaches de race Tharparkar était traité de la façon suivante : traite pratiquée 4 fois par jour, et massages de la mamelle 15 à 20 jours avant la mise bas; un autre lot était seulement trait 2 fois par jour. Dans le premier lot, le rendement dépassait de $47 \%$ celui du second (Parr et Sen, 1947).

A la base de l'amélioration en quantité et qualité, chez les races indigènes qui, le climat ou les facteurs humains s'y opposant, ne peuvent subir le croisement avec des géniteurs européens, il doit donc y. avoir la sélection, l'alimentation, l'entretien, la gymnastique fonctionnelle. Tenant compte des résultats obtenus chez des vaches indigènes du Kenya par la seule hygiène alimentaire et l'entretien, Daubney concluait, dès 1938, qu'on pourrait obtenir par ces moyens des résultats qu'on n'eût pas escomptés auparavant. Avec une telle production. et compte tenu de la résistance des races locales, il lui apparaissait superflu et hasardeux de tenter l'acclimatement de races européennes sur une large échelle, tant que l'état sanitaire n'est pas satisfaisant.

Il est intéressant de retenir l'intérêt que pourrait avoir le recours aux œstrogènes de synthèse. On a pu faire observer (Balay, 1949) qu'on pourrait tenter de provoquer la lactation chez les femelles stériles, nombreuses en Afrique, pratique qui, d'ailleurs, serait onéreuse en élevage extensif.

L'aspect du problème est différent là où apparait dès maintenant, la possibilité d'une amélioration par le croisement avec des races importées; pour disparates que soient les résultats, il en est quand même qui portent leçon et peuvent éviter des déboires; tel est le cas de la valeur amélioratrice des races de Bos indicus, et particulièrement des zébus de l'Inde, et la particulière résistance de la race de Jersey; ce sont ces exemples qui doivent aider à la constitution de sous-races résistantes, à la formation d'un cheptel laitier résistant dans les régions où le permet le facteur humain.

Quant à la création de noyaux purs de races européennes, elle reste de possibilité limitee; mais sans doute sera-t-elle néanmoins une solution dans certaines régions subtropicales et aussi pourra-t-elle contribuer au ravitaillement des villes par de petits troupeaux laitiers, entretenus dans des conditions artificielles qui combattent les facteurs nocifs du climat.

\section{BIBLIOGRAPHIE}

1948. BAKALOR (S.). - Recherches sur la composition du lait sud-africain. Farm. in $\mathrm{S}$. Africa, 23, 271

1949. BALAY (R.). - Ia pratique de la lactation provoquée par l'emploi des cestrogènes de synthèse. Bullet. Serv. Clev. Afr. Occid. fr., $\square, 59$.

1940. BONSMA (J.-C.). - The influence of climatological factors on Catte. Farming in S. Africa, 373.

1942. CACCIAGUERRA OJ ). - La production laitière en Algéxie. Semaine du lait, 4, 48.

1945. CAMERON (R.-H.). - Progrès du bétail indigène du centre vétérinaire d'élevage de Sangalo. East afric. agric. Journ., 11, 20.

1948. CARNEIRO .(G.-G.) et LUSH (J.). - Variations in yield of milk onder the penkeeping system in Brazil. Journ. Dairy Sc.,31, 203.

1933. CURASSON (G.). - Note sur la composition du lait des vaches africaines et son ntilisation dans l'alimentation. Bullet. Soc. Patho. exot., 26, 536.

1948. CURASSON (G.). I Le climat tropical et la production animale. Acta tropica, $5,97$.

1942. DAUBNEY (F,). - Suitability of the White highlands of Kenya for grade cattle of Enropean breeds. East. Afric. agric. Journ.. 7, 127.

1947. DAVIS (R.-N.) HARLAND (F.-G.), CASTER (A.-B.) et KELLNER (R.-H.). - Variations in the constituents of milk under Erizona conditions. Journ. Dairy Sc., 30, $415,425,435$

1948. DOUGLAS (H.-K.), LEE et PHILLIPS( R.-W.). - Assessment of the adaptability of livestock to climatic stress. Journ. of anm. Sc., 7, 291.

1937. DUFOUR (V.). - Etude sur les laits consommés à Dakar. Ann. méd. et pharmacie colon., 35, 87.

1932. EDWARD (J.). - Breeding for milk production in the tropics. Journ. dairy Res., 3, 281.

1931. ELS. - Enalyse du lait du bétail indigène de l'Urandi Bullet, agric. Congo belge, 292.

1945. GAALAAS (R.-F.). - Effect of atmospheric temperature on body temperature and respiration rate of Jersey cattle. Journ. dairy Sc., 28, 555.

1949. GIRARD (M.). - La race bovine de Meknès. Rev. élev. et rnédec. vét. pays tropic., 3, 53.

1945. GUY (G.). - La production laitière en Indochine. Thèse Altort, Paris.

1932. HAMMOND (J.). - Report in cattle breeding in Jamaica and Trinidad. Empire Marketing Board Bullet., no 58 .

1892. HILIS (G.-L). - The effects of weather upon the quality and quantity of millk. Vermont Agric. Exper. Stat., 128.

1946. HOWE (J.-W.), - The effects of varying amounts of zebu blood of the adaptability of dairy cattle to conditions in Jamaica. lowa State College Thesis Iowa.

19.17. ITTNER (N.-R.). - A progress report on livestock investigations in the Imperial Valley. Univ. of Calif. agric. experim. Stat.

1939. JACOBSEN (D.-H.) et WALTERS (G.-C.). - Factors affecting the composition of milk. South Dak. Stat. Bullet. $n \circ 331$ 
1947-48 JAUFFRET (M.) el AUTRET (M.) .-.- Les laits et la production laitière àu Tonkin. Revi Elev, des pays tropir., 1, $201 ; 2,5$.

1945. JORDAO (L.-P.) et ASSIS (F, de P.), - Contribuiçao para o studo do gado Holandès. Bol. 'Indust. anim. Soc Paulo, 8, 1-2.

1948. KANNAN (A.) et BÁSU (K.-P), - La phosphałase du lajt. Ind. Journ. Dairy $S c, 1,16$.

1937. KAY (H.-D.). - The biochemistry of milk secretion.. Journ. Royal Soc. of Arts; 86, 841 .

1946. LABARTHE (C,-A.), - La variacion estacional de la materia grasa de la leche en algunas zonas de la Republica Argentina. Rev. Fac. agron. B. Aires, 11, $18 \mathrm{I}$.

1949. IAMIRE., - La race hollandaise au Maroc. Rev. élev. et Méd. vétér. pays tropic., 3,5I.

1946. LAZARUS (A.-J.) - Le buffle comme animal laitier Indian Farming, 7, 247 .

1932. LESSER (J.). - - Milk deficiénce in solids non fat. Journ. of Minist. of agric., 39, 340 .

1930. Ј $\triangle 7 . \overline{C H}$. - Enquête sux les laits de vache de la région de Hanoi. Bull. Soc. Médicó-chirurg. de $1^{\dagger}$ Indóchine 2, $81 \hat{\text { a. }}$

1946. MAHAMOOTH (T.-M.-Z.). - Mesures adoptées et envisagée's pour une production accrue du lait dans l'île de Ceylan. Tropic. Agric., 102, 41.

1946. MILILER (W.-C.).' - Industrie animale, alimentation, élevage et Service vétérinaire dans les Indes Occidentales. Bull. Devel. Welfare West Indies, n ${ }^{\circ} 19$.

19,45. MINETT (F.-C.) et SEN (S.). - Rectal temperatures of certain animals at rest. Ind. Journ. of Vet. Sc. 15, 62.

1947. MINETT (F.-C.). - Effects of artificial showers, natural rains and wallowing on the body temperatures of animals. Journ. of anim. SC, 5,147 .

1948. MORNET .(P.) et GILBERT (Y). - Rapport sur la production et l'utilisation, du lait, en Afrique occidentale française. Bullet. Serv. Elevage A.O.F., 1, 59.

1947. PAR (C.-H.) et SEN (S.).' - Effects of four times milking and handling on the yield of cows of the Tharparkar breed. Ind, Journ. Vet. Sc., 17, 75 .

1948. PHILIPPS RALPH (W.). - Breeding livestock adapted to unfavorable environments. F.A.O. Agric. Studies, no 1 , Washington.

1942. PRIGENT (R.), KA'NE (P.) et KÁ (B.). - Elevage cu bouf en Mauritanie. Bullet. Serv. Zoot. et Epiz. A.O.F., 5, 235.

1946. PURCHASE (H.-P.) et REVERBERI (A.-H.). - Les constituants de la matière sèche des laits du Kenya. East afric. Agric. Journ., 12, 50.
1947. RCES (H.-V.). - Seasonal variations in the solid not fat and on fat content in maxket milk. Austr. Journ. 'Dairy Technol., 2; 3.

1946. VAN RENSBURG (S.-W.-J.). - Faible tı neux en matière sèche non grasse du lait en Afrique ly Sud. Farm. in $S$. Africa, 21, 217.

1947. VAN RENSBURG (S,-W,-J). - The s scr etion of abnormal milk by quaters free from IKnown pathogens. Onderstepoort Journ.'; 22, 91

1938. REGAN (W.-M) et RICHARDSON' (G.-A.). - Reactions of the dairy cow to changer of enviromental temperature. Journ. dairy Sc., 21, 73 .

1944. RHOAD (A,-O.). - The Iberia heat tolerance test for cattle. Tropic. Agric., 21, I62.

1948. RIEK (R.-F.) et DOUGLAS (H.-K. Lee). - Reactions to hot atmospheres of Jersey cows in milk. Journ. of dairy Res., 15, 219

1941. RIDDET (N.), CAMPBELL (I.-L.), McDOWALL (F.-H.) et COX (G.-A.) - The relation of plane of nutrition to milk production and mille composition in New Zealand. New Zeal. Journ. Sc. and Technol., 23, 80.

1949. SAILLARD $\left(M_{1}\right)$, - Le métissage tarentais en Efxique du Nord. Rev. Elev. et médec. Vét. pays trop., 3, 511

1948. SANDERS (R. N.). - The adverse effeot of cool weather on mille production on the tropics. Agric. Journ. 'Fidji, 19, 39:

1947. SEATH (D.-M.) et MLLER (G.-D.). - Heat tolerance comparison between Jersey and Holstein cows. Journ, of anim. Sc., 6, 24.

1948. STAMFORTH (A.-R.). - Elevage laitier dans la zone à glossines du.Soudan anglo-égyptien. East' Afric. Journ., 13, ZZZ4.

1947. SINHA (K,-C.) et MINETT (F,-C,). - Application of water to the body suxface of bufialoes and its effect on milk yield. Journ. anim. Sc, 6, 258.

1941. SMITH (J.-A.-B.). — Climate and milk production. Journ. Dairy Res., 12, 100.

1936. TURNER (C.-V.). - Factors affecting the composition of milk. Miss: agrıc. exper. Stat, $n^{\mathrm{e}} 365$.

1939. VILIEGAS (V.). - Pxoduction animale en Cochinchine, Cambodge, Siam et Malaisie. Philip. agricult., 27, 693.

1938. TRAMBICUS $(J)$. - Einfluss der' jahreszeit auf die zusammensetzung der milch. Milchwirtsch. Firsch, 19, 353

1945. WRIGHT, NORMAN (C.). - Development of cattle breeding and milk production in Ceylan. Brtish colonial Office, Eastern, no 179. 